\title{
A NEW APPROACH TO ESTIMATE PEDESTRIAN DELAY AT SIGNALIZED INTERSECTIONS
}

\author{
Sankaran Marisamynathan, Perumal Vedagiri \\ Dept of Civil Engineering, Indian Institute of Technology Bombay, India
}

Submitted 1 November 2014; resubmitted 23 January 2015, 24 April 2015; accepted 10 June 2015;

published online 29 March 2016

\begin{abstract}
Enhancing pedestrian safety and improving the design standards of pedestrian facilities at signalized intersection requires a clear understanding of pedestrian delay model and pedestrian crossing behaviours under mixed traffic condition. The existing delay models do not consider the behavioural constrains of pedestrians. This research has been undertaken with the aim of developing a suitable pedestrian delay model for signalized intersection crosswalks, based on considering actual pedestrian crossing behaviours. The required model parameters were extracted from the video-graphic survey conducted for the selected four signalized intersections in Mumbai (India). Crossing behaviours of pedestrians were examined through field data in terms of pedestrian arrival pattern, crossing speed, compliance behaviour and pedestrian-vehicular interactions. Based on pedestrian crossing behaviour analysis results, two new pedestrian delay estimation models were developed and the models were validated by comparing with field and existing model values. The performance level of the proposed models is showing more precise and reliable solutions. The first pedestrian delay model is developed on the basis of compliance behaviour, has two components, such as waiting time delay and crossing time delay. This model can be used to evaluate pedestrian Level Of Service (LOS) and signal timing optimization. The second developed pedestrian delay model is based on noncompliance behaviour, has three components, such as waiting time delay, crossing time delay, and pedestrian-vehicular interaction delay. This model can also be used to evaluate the quality of pedestrian flow, estimating accurate pedestrian delay and LOS for local conditions, which is representative of the prevailing pedestrian condition.
\end{abstract}

Keywords: pedestrian delay; signalized intersection; crossing speed; compliance behaviour; arrival pattern; interactions.

\section{Introduction}

Walking is still considered as a vital travel mode, though there have been many modes of motorized transportations. Significant attention is being given to walking, due to large amounts of pollution and congestion attributed to motorized transportations. To increase number of walking trips, there is a need for clear understanding of pedestrian behaviour under various conditions. Among various pedestrian facilities, signalized intersections are complex and high risk for pedestrians because pedestrians and vehicles are sharing the same road space in terms of crosswalks at signalized intersections. Various types of pedestrian control strategies, such as fixed time control, dynamic control, coordinated control and adaptive control are provided at signalized intersections based on pedestrian and traffic flow and geometric conditions. Fixed-time control signal is the most cost effective among all the strategies and is widely used at signalized intersections under mixed traffic conditions.
A fixed time control for highly populated areas needs to be reconsidered since the flow of pedestrian traffic varies largely. Adaptive traffic control or optimization is required for better traffic control in such signalized intersections. The former needs complete rearrangement of the infrastructure of traffic control signals while the latter can be utilized with existing design.

Delay is the key parameter for signal timing optimization and Level Of Service (LOS) assessment at signalized intersections. It is essential to estimate delay more precisely with due consideration of all possible traffic parameters. Recently, majority of the studies have been focused on vehicle delay estimations and not on pedestrian delay estimations (Olszewski 1993; Mousa 2002). Highway Capacity Manual (HCM 2010), the most referred manual estimates pedestrian delay on the basis of signal cycle length and red phase duration with the assumptions of uniform pedestrian arrival rate, pedestrian compliance with signal, no variation in crossing

Corresponding author: Sankaran Marisamynathan

E-mail: marisamy1989@gmail.com 
speed and no interaction between pedestrian and vehicle. These assumptions are not suitable for developing countries under mixed traffic conditions where the pedestrians' behaviour is highly different and thus the model proposed by HCM (2010) is inefficient to estimate pedestrian delay at signalized intersections in India. Pedestrian delay at signalized intersection is difficult to estimate accurately because of pedestrian crossing behaviour and vehicular interactions. Hence, there is a need to develop pedestrian delay model, which is representative of the prevailing pedestrian situation for local conditions prevailing in India. The results of this study may be useful in determining LOS, signal timing optimization, evaluating the quality of pedestrian flow, providing safe and adequate crosswalk for local conditions, where pedestrians noncompliance are predominant.

This paper is organized as follows: Firstly, the existing studies on pedestrian crossing behaviours and pedestrian delay models with their limitations are discussed. Secondly, study objective, methodology and locations' details have been described. Thirdly, pedestrian crossing behaviours are analysed. This is followed by pedestrian delay models that have been developed using this analysis. Validation is then performed by comparing results from the proposed models with field values and existing model values. Finally, conclusions and recommendations for future studies are provided.

\section{Review of Earlier Studies}

\subsection{Pedestrian Crossing Behaviour}

Accurate estimation of pedestrian delay at signalized intersection is inherently complex because of varying pedestrian crossing behaviours, such as arrival pattern, crossing speed, noncompliance, and pedestrian-vehicular interaction under mixed traffic conditions. Major reason for failure of all existing models in highly populous city, such as Mumbai in India is to consider constant pedestrian crossing speed. This constant value of pedestrian crossing speed is not applicable for varying pedestrian crossing behaviour in India. The most referred recent Indian manual does not consider pedestrian crossing behaviour variation and delay model at signalized intersections (IRC: 103-2012). Most of the studies on crossing speed variations consider only sidewalks (Al-Azzawi, Raeside 2008; Rastogi et al. 2011).

Very few studies have identified crossing speed variations at crosswalks in signalized intersections (Gates et al. 2006; Huang, Ma 2010). Most of the existing studies assumed non-uniform pedestrian arrival for pedestrian delay estimation (Li et al. 2005b; Yang et al. 2005; Xie et al. 2012; Nagraj, Vedagiri 2013) and also fail to estimate accurate pedestrian delay value at signalized intersections. Commonly, pedestrians are prohibited to enter crosswalk during flashing red and red phases. In developing countries like India, traffic conditions and pedestrian signal system are highly complex and pedestrians have been found to enter crosswalk to reduce waiting time delay during non-green phases. Some of the reasons of pedestrian noncompliance are mixed traf- fic conditions, low quality traffic management, pedestrian characteristics and complex signal system (Virkler 1998; Li et al. 2009; Chen et al. 2011; Jiang et al. 2011; Zhou et al. 2011). Research works carried out on pedestrian delay due to pedestrian-vehicular interactions in crosswalks are very less (Ling et al. 2012; Xie et al. 2012) and most of the studies have ignored pedestrian delay received during green phases by vehicle interaction on crosswalks (Li et al. 2005a). Therefore, in this paper mixed traffic conditions and pedestrian crossing behaviour are considered and proposed a new methodology to estimate pedestrian delay at signalized intersection.

\subsection{Existing Pedestrian Delay Models}

At present, HCM's (2010) pedestrian delay model is the most commonly used model to estimate pedestrian delay value at signalized intersections:

$$
d_{p}=\frac{\left(C-G_{\text {Walk,mi }}\right)^{2}}{2 \cdot C},
$$

where: $d_{p}$ is the average pedestrian delay; $C$ is the cycle length; $G_{W a l k, m i}$ is the effective walk time for the phase serving the minor street through movement. The model is developed considering assumptions such, as uniform arrival rate of pedestrian, pedestrian comply with traffic signal, and fixed cycle length with the delay being dependent only on signal time. It is to be noted that these assumptions are not suitable for mixed traffic conditions prevailing on Indian roads. An alternative model (Braun, Roddin 1978) was developed by considering pedestrian noncompliance behaviour with respect to signal timings and the model is as follows:

$$
d=\frac{F \cdot(C-G)^{2}}{2 \cdot C},
$$

where: $F$ is the fraction of pedestrians who arrive during non-green phases and comply with traffic signals. This model assumes that the noncompliant pedestrian crosses the crosswalk without receiving any delay, which is not the scenario in reality where numbers of noncompliance pedestrians interact with vehicle and receive delay in crosswalk. Pedestrian-vehicle interaction delay has not been taken into account in this model. It was also identified that most pedestrians reduce the delay by noncomplying during clearance time (Virkler 1998) and the model developed with this into account is as follows:

$$
d=\frac{(C-(G+0.69 \cdot A))^{2}}{2 \cdot C},
$$

where: $A$ is pedestrian clearance time. This model has considered that $69 \%$ of pedestrians do not comply with traffic rules during clearance time thus making it inapplicable for all type of traffic conditions. Based on maximum pedestrian crossing rate, pedestrian delay model was developed (Oh, Sisiopiku 2000) and correction factor for arrival rate was calculated by using queuing theory. In this model, arrival pattern was not analysed with any distribution studies and model estimates only waiting time delay. Another delay model was developed 
based on pedestrian arrival pattern and noncompliance rate (Li et al. 2005b; Yang et al. 2005), which is as follows:

$$
d=d_{G}+\frac{k_{N U} \cdot k \cdot R_{E}^{2}}{2 \cdot C}
$$

where: $d_{G}$ is the average delay of pedestrians arriving during green phases; $k_{N U}$ is the adjustment factor for non-uniform arrival rate; $k$ is the absolute value of the decreasing line's slope during non-green phase; $R_{E}$ is effective red time. This model does not considered interaction delay during pedestrian non-green phases. Correction factor used in this model's non-uniform arrival pattern has been made without performing statistical fitting tests. The correction factor used in this model thus fails to accurately estimate pedestrian delay and is also not applicable for high pedestrian flow scenarios. The same author (Li et al. 2005) used Monte Carlo method to estimate pedestrian delay based on vehicle generator and pedestrian generator. Pedestrian arrival rate was assumed to satisfy exponential distribution without any field data analysis to support and also has considered pedestrian noncompliance rate without interaction delay.

Based on pedestrian arrival, a pedestrian delay model was developed (Kruszyna et al. 2006), which considered the signal duration and arrival flow parameters for model development and neglected pedestrian behaviour. An improved pedestrian delay model was developed with a two stage crossing design (Wang, Tian 2010), which is a modified form of HCM (2010) waiting time delay model and considered the same assumptions put forth in HCM (2010) pedestrian delay model. Simultaneously another delay model was constructed with pedestrian gathering and dissipating characteristics (Chen et al. 2010). The model was compared with simulated results generated by VISSIM but the model was not validated with field data and also did not consider pedestrian crossing behaviour. The model was lacking generality and provided inaccurate delay value.

Recently, a delay model was developed by considering pedestrian arrival pattern and compliance rate $(\mathrm{Na}-$ graj, Vedagiri 2013) and it is as follows:

$$
d=D_{g}+\frac{K_{c} \cdot K_{n u} \cdot(C-G)^{2}}{2 \cdot C},
$$

where: $D_{g}$ is the time difference between ideal time taken to cross and actual time; $K_{c} \cdot K_{n u}$ is the factor showing compliance and non-uniform arrival rate. This model uses an arrival pattern, same as in Li et al. (2005b) studies, without distribution testing tests. Crossing time delay used in this paper was measured from field. Hence, review of existing pedestrian delay models show that the existing models might not be able to accurately estimate pedestrian delay values under mixed traffic conditions because of not considering pedestrian crossing behaviour. There is a need for a pedestrian delay model that incorporates pedestrian crossing behaviour characteristics and vehicular interactions under mixed traffic condition. This study is an attempt in this direction.

\section{Study Objective}

The objective of this paper is to develop suitable pedestrian delay estimation model at signalized intersections by considering pedestrian crossing behaviour and vehicular interactions under mixed traffic conditions. In view of this, crossing behaviour of pedestrians, such as pedestrian arrival pattern, crossing speed variation, pedestrian noncompliance, and pedestrian-vehicular interactions at crosswalk were analysed. Two different delay models have been developed to incorporate pedestrian behavioural characteristics.

\section{Methodology}

Two sets of data have been collected from the selected four signalized intersections. One set of data consists of pedestrian compliance with traffic signal and other consists of pedestrian noncompliance with signal and also pedestrian-vehicular interactions. With the data existing of pedestrian compliance with signal, first pedestrian delay model has been proposed and it has followed two components: (i) the average waiting time delay, based on modified HCM (2010) model by considering pedestrian non-uniform arrival rate and (ii) the crossing time delay, based on crossing speed variations. The data consisting of pedestrian noncompliance with signal and pedestrian-vehicular interaction is used to develop the second delay model and it has followed three components: (i) average waiting time delay, based on modified HCM (2010) model by considering pedestrian noncompliance rate, (ii) the crossing time delay, and (iii) the pedestrian-vehicular interaction delay, based on developed Binary Logit (BL) model. The proposed models have been validated using field data and compared with existing delay models.

\section{Data Collection}

Data were collected from the highly populous metropolitan city, Mumbai (India). Data collection duration and number of crosswalks were fixed on the basis of existing studies. In existing studies, the selected number of crosswalks varies from one to fourteen. Inference from literature studies, approximately six to ten crosswalks are suitable for analysing pedestrian crossing behaviour and model development (Li et al. 2005b; Yang et al. 2005; Kruszyna et al. 2006; Huang, Ma 2010; Nagraj, Vedagiri 2013). The study locations are Holkar junction (A), Mahim junction (B), Samaj junction (C) and Vivekanand junction (D), which are located at the central part of Mumbai city and details of selected six crosswalks are shown in Figure.

Signalized intersection sites chosen were of a typical four-arm type with fixed traffic signal cycle lengths. Based on pedestrian flow and geometric characteristics, the major and minor crosswalks were selected at location $\mathrm{A}$ and $\mathrm{B}$ and major crosswalks were selected at locations $\mathrm{C}$ and $\mathrm{D}$ for study purpose. The observed field data for bi-directional pedestrian flow and vehicle volume from the selected sites are shown in Table 1. 


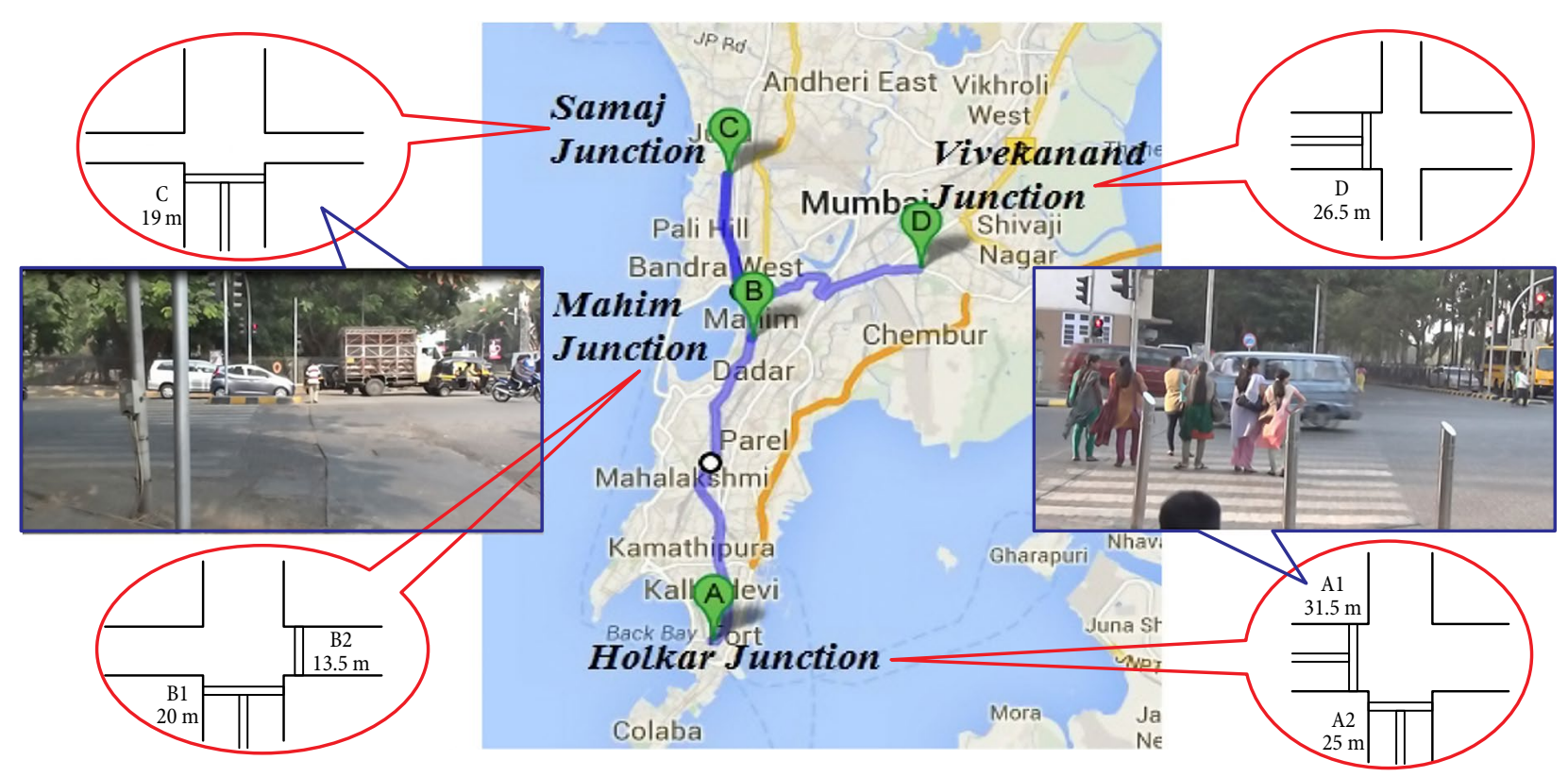

Fig. Selected study location in Mumbai (India)

Table 1. Study location and field observed data

\begin{tabular}{|c|c|c|c|c|c|c|c|c|c|c|}
\hline \multirow[b]{2}{*}{ Intersection name } & \multirow{2}{*}{$\begin{array}{c}\text { Cross } \\
\text { walk } \\
\text { identity }\end{array}$} & \multirow{2}{*}{$\begin{array}{c}\text { Crosswalk } \\
\text { flow } \\
\text { [ped/h] }\end{array}$} & \multirow{2}{*}{$\begin{array}{l}\text { Vehicle } \\
\text { volume } \\
{[\mathrm{pcu} / \mathrm{hr}]}\end{array}$} & \multirow{2}{*}{$\begin{array}{l}\text { Date of } \\
\text { survey }\end{array}$} & \multirow{2}{*}{$\begin{array}{l}\text { Time of } \\
\text { survey }\end{array}$} & \multirow{2}{*}{$\begin{array}{l}\text { Crosswalk } \\
\text { length }[\mathrm{m}]\end{array}$} & \multicolumn{4}{|c|}{ Pedestrian phase [s] } \\
\hline & & & & & & & Green & $\begin{array}{c}\text { Flashing } \\
\text { red }\end{array}$ & Red & $\begin{array}{l}\text { Cycle } \\
\text { time }\end{array}$ \\
\hline \multirow{2}{*}{ Holkar junction } & $\mathrm{A} 1$ & 337 & 3779 & 4.12 .14 & $5-6 \mathrm{pm}$ & 31.5 & 25 & 4 & 121 & 150 \\
\hline & $\mathrm{A} 2$ & 83 & 2311 & 5.12 .14 & $5-6 \mathrm{pm}$ & 25 & 22 & 3 & 55 & 80 \\
\hline \multirow{2}{*}{ Mahim junction } & $\mathrm{B} 1$ & 402 & 3308 & 5.12 .14 & $9-10 \mathrm{am}$ & 20 & 35 & 2 & 106 & 143 \\
\hline & B2 & 272 & 1081 & 5.12 .14 & $9-10 \mathrm{am}$ & 13.5 & 19 & 3 & 121 & 143 \\
\hline Samaj junction & $\mathrm{C}$ & 147 & 3794 & 4.12 .14 & $9-10 \mathrm{am}$ & 19 & 12 & 3 & 115 & 130 \\
\hline $\begin{array}{l}\text { Vivekanand } \\
\text { junction }\end{array}$ & $\mathrm{D}$ & 166 & 5567 & 6.12 .14 & $9-10 \mathrm{am}$ & 26.5 & 21 & 4 & 60 & 85 \\
\hline
\end{tabular}

The percentage composition of two wheelers, auto-rickshaws, cars, buses, trucks, LCVs and bi-cycles are 12, 19, $54,8,2,3$ and 1 , respectively from all locations.

Data were collected using a video recording device during peak hour. From the video recording, information about pedestrian volumes, crossing time, crosswalk length, crossing locations (whether using the crosswalk or not using), pedestrian phase time (whether in green phase, flashing red phase and red phase), crossing behaviours (such as walking or running, alone or platoon and walking speed), pedestrian appearance character (like gender and age group by visual appearance) and pedestrian-vehicle interactions were collected. Using ALLCapture software ( $h t t p: / / w w w$.allcapture.com), the required pedestrian sample data were extracted.

\section{Pedestrian Crossing Behaviour Analysis}

At present, there is no proper pedestrian crossing behaviour model at signalized intersections under mixed traffic conditions. An attempt has been made in this paper to analyse pedestrian crossing behaviour by considering actual field conditions, like pedestrian arrival pat- tern, crossing speed, possible noncompliance behaviour of pedestrians, and pedestrian-vehicle interaction on crosswalk from field observed data.

\subsection{Pedestrian Arrival Pattern}

HCM (2010) assumed that pedestrians arrival pattern follows uniform pattern and pedestrian delay model was developed at signalized intersections. But the assumption is not suitable for all conditions and it depends on pedestrian arriving volume at signalized intersections (Marisamynathan, Vedagiri 2013). In existing studies, pedestrian signal time was divided into several equal sub-phases for one hour and arrival pattern were tested and it clearly shows that the arrival pattern follow a non-uniform arrival pattern at Mumbai (India) (Marisamynathan, Vedagiri 2013). Pedestrian arrival pattern has been analysed from both directions of pedestrian crossing movements and totally 12 directional movements were examined from 6 crosswalks for suitable distribution fitting by using the Minitab 16 software (http://www.minitab.com). It was observed from the results that, if the pedestrian arrival rate per direction is 
less than $80 \mathrm{ped} / \mathrm{hr}$, it follows Poisson distribution and if the pedestrian arrival rate per direction is greater than $80 \mathrm{ped} / \mathrm{hr}$, then it follows negative binomial distribution. The reason the data do not follow uniform distribution is due to the crossing of pedestrians in platoon rather than as individuals and bunching of arrivals.

The assumption of uniform arrival pattern fails to estimate accurate delay and the correction factor for pedestrian non-uniform arrival pattern was introduced based on field data with respect to pedestrian arriving volume. The correction factor for non-uniform arrival pattern is the average of the ratio between actual pedestrian arrival rate $Q$ at sub-phase interval $i$, and uniform pedestrian arrival rate $q$ at the same sub-phase interval. The correction factor is as follows:

$$
\alpha_{1}=\frac{1}{n} \cdot \sum_{i=1}^{n} \frac{Q_{i}}{q_{i}} .
$$

The linear relationship between $\alpha_{1}$ and number of pedestrians arriving during non-green phase per hour $V$ on crosswalk of signalized intersections was developed from field data and it can be expressed by:

$$
\alpha_{1}=0.002 \cdot V+0.734, R^{2}=0.86 \text {. }
$$

The correction factor is applicable for all types of signalized intersections with mixed traffic conditions. Waiting time delay can be estimated accurately with the use of the above mentioned correction factor.

\subsection{Pedestrian Crossing Speed}

In India, the present design practice in estimating pedestrian delay at signalized intersection is based on the assumption that constant pedestrian walking speed is equal to $0.95 \mathrm{~m} / \mathrm{s}$ (IRC: 103-2012). The field study conducted in Mumbai shows that the crossing speed of pedestrians varies largely from the assumed constant value. Crossing speed is defined as the crossing distance divided by crossing time. Two lines were drawn in starting and ending point of the crosswalk and pedestrian foot moments (start to end) were followed individually to estimate the crossing time of particular pedestrian at crosswalk by using ALLCapture software.
ANOVA test and Student's $t$-test were performed to identify the influencing factors for crossing speed variations at $99 \%$ confidence interval by using SPSS 16.0 (http://www.ibm.com/software/analytics/spss). The main factors analysed in statistical test were pedestrian gender, age group, number of pedestrian, traffic signal, and using crosswalk marking. Statistical test results are shown in Table 2. Statistics indicate that pedestrian gender, age group, and number of pedestrian in-group are the significant factors for pedestrian crossing speed variations.

From Table 2, the following inferences are made. The average crossing speed of a male pedestrian $(1.34 \mathrm{~m} / \mathrm{s})$ is more than a female pedestrian $(1.26 \mathrm{~m} / \mathrm{s})$. As expected, adult pedestrian (of age from 18 years to 60 years) crossing speed $(1.34 \mathrm{~m} / \mathrm{s})$ is more than that of an old pedestrian (of age $>60$ years) crossing speed $(1.21 \mathrm{~m} / \mathrm{s}$ ). No major variation between Green phase crossing speed $(1.34 \mathrm{~m} / \mathrm{s})$ and red phase crossing speed $(1.31 \mathrm{~m} / \mathrm{s})$. Platoon or group of pedestrians crossing speed $(1.21 \mathrm{~m} / \mathrm{s})$ is less than that of a single pedestrian crossing speed $(1.38 \mathrm{~m} / \mathrm{s})$.

The 15th and 85th percentile crossing speed is varying from $0.95-1.12 \mathrm{~m} / \mathrm{s}$ and $1.43-1.66 \mathrm{~m} / \mathrm{s}$ respectively. Crossing time delay can be estimated accurately with the use of the above mentioned 15th percentile crossing speed.

The field value $(1.34 \mathrm{~m} / \mathrm{s})$ was found to be nearly equal to field value of $1.33 \mathrm{~m} / \mathrm{s}$ measured in China ( $\mathrm{Li}$ et al. 2005b; Yang et al. 2005) and also compared with other international crossing speed values of $1.21 \mathrm{~m} / \mathrm{s}$ for US (Gates et al. 2006), $1.15 \mathrm{~m} / \mathrm{s}$ for Malaysia (Rahman et al. 2012), $1.45 \mathrm{~m} / \mathrm{s}$ for Hong Kong (Lam et al. 2002) and $1.8 \mathrm{~m} / \mathrm{s}$ for London.

\subsection{Pedestrian Compliance Behaviour}

From the field observed data, it was observed that, out of 1407 pedestrians sampled, there were a total of 806 pedestrians who have non-compliance with traffic signal and crossed during the red phase. During pedestrian non-green phases, arriving pedestrians attempt to cross the crosswalk by finding suitable gap from vehicles

\begin{tabular}{|c|c|c|c|c|c|c|c|}
\hline Factor & Stage & Mean $[\mathrm{m} / \mathrm{s}]$ & Std $\operatorname{dev}[\mathrm{m} / \mathrm{s}]$ & Sum of squares & $F$-value & $P$-value & $t$-value \\
\hline \multirow{2}{*}{ Gender } & male & 1.343 & 0.328 & \multirow{2}{*}{122.02} & \multirow{2}{*}{$13.389^{\mathrm{a}}$} & \multirow{2}{*}{$0.000^{\mathrm{a}}$} & \multirow{2}{*}{$3.659^{\mathrm{a}}$} \\
\hline & female & 1.260 & 0.235 & & & & \\
\hline \multirow{2}{*}{ Age group ${ }^{*}$} & adult & 1.344 & 0.315 & \multirow{2}{*}{122.02} & \multirow{2}{*}{$25.247^{\mathrm{a}}$} & \multirow{2}{*}{$0.000^{\mathrm{a}}$} & \multirow{2}{*}{$5.025^{\mathrm{a}}$} \\
\hline & old & 1.214 & 0.279 & & & & \\
\hline \multirow{2}{*}{$\begin{array}{l}\text { No of } \\
\text { pedestrian }\end{array}$} & single & 1.387 & 0.327 & \multirow{2}{*}{122.02} & \multirow{2}{*}{$90.594^{\mathrm{a}}$} & \multirow{2}{*}{$0.000^{\mathrm{a}}$} & \multirow{2}{*}{$9.518^{\mathrm{a}}$} \\
\hline & platoon & 1.216 & 0.253 & & & & \\
\hline \multirow{2}{*}{$\begin{array}{l}\text { Traffic } \\
\text { signal }\end{array}$} & red & 1.313 & 0.267 & \multirow{2}{*}{122.02} & \multirow{2}{*}{$3.658^{\mathrm{b}}$} & \multirow{2}{*}{$0.056^{\mathrm{b}}$} & \multirow{2}{*}{$-1.912^{\mathrm{b}}$} \\
\hline & green & 1.348 & 0.371 & & & & \\
\hline \multirow{2}{*}{$\begin{array}{l}\text { Crosswalk } \\
\text { mark }\end{array}$} & walking in the marking & 1.336 & 0.319 & \multirow{2}{*}{74.79} & \multirow{2}{*}{$0.517^{\mathrm{b}}$} & \multirow{2}{*}{$0.473^{\mathrm{b}}$} & \multirow{2}{*}{$-0.719^{\mathrm{b}}$} \\
\hline & not walking in the marking & 1.350 & 0.272 & & & & \\
\hline
\end{tabular}

Table 2. Statistical details of crossing speed

Notes: ${ }^{a}$ value is significant; ${ }^{b}$ value is not significant (at the $99 \%$ of confidence level); ${ }^{*}$ children age group data sample is not sufficient for modelling. 
across each lane in the crosswalk. Reasons for pedestrians' noncomplying with traffic signal were to reduce more waiting time delay, less time for pedestrian green phase and less vehicle flow, where pedestrians get suitable vehicular gap.

Pearson's correlation test was performed to identify the factors influencing pedestrian noncompliance behaviour at $95 \%$ confidence interval. The main factors analysed in statistical test were pedestrian gender, age group, number of pedestrian, direction of movement, crossing speed and using crosswalk marking. Of the main effects, the most significant were: crosswalk marking $(R=0.177, p=0.000)$, gender $(R=-0.113, p=0.001)$, and group size $(R=0.070, p=0.042)$. The following inferences were made from the statistical results. Male pedestrians were more likely to be noncompliant with traffic signal. It the crosswalk marking is present, most of the arriving pedestrians comply with traffic signal.

According to the observations, the average proportion of pedestrians non-complying with the traffic signal is found to be $57.29 \%$ in the selected sites. Average pedestrian non-compliance in Mumbai has also been compared with results from studies performed by Virkler (1998) (69\% for Columbia) and Li et al. (2005b) (67\% for China). It is inferred, that in developing countries, the average pedestrian noncompliance rates are more or less the same, which requires special attention and modification in pedestrian delay estimation process. These modification models can give truer picture on pedestrian delay at intersections and also useful to take suitable corrective measures to enhance safety at signalized intersections.

\subsection{Pedestrian-Vehicular Interaction}

Pedestrian-vehicular intersection delay is defined as 'extra crossing time taken by pedestrian due to vehicu- lar interaction with pedestrian at crosswalk during pedestrian green and non-green phase.' During pedestrian green phase or vehicle flashing green phase, vehicle interactions occur with pedestrians due to driver's noncompliance behaviour. During pedestrian non-green phase or pedestrian flashing green phase, pedestrian interactions occur with vehicle due to pedestrian noncompliance behaviour.

Sometimes drivers stopped the vehicles or reduced the vehicle speed and give way to noncompliant pedestrians. According to the above explanation, there are only two possible outcomes: pedestrian receives either delay or not due to vehicular interaction in crosswalk. Therefore, a BL model was considered to find the probability of interactions between pedestrian and vehicle.

$$
\begin{aligned}
& P\left(y_{n}\right)=\frac{e^{x}}{1+e^{x}} ; \\
& X=\beta_{0}+\beta_{1} \cdot x_{1}+\beta_{2} \cdot x_{2}+\ldots+\beta_{p} \cdot x_{p},
\end{aligned}
$$

where: $P\left(y_{n}\right)$ is probability that pedestrian $n$ receive interaction delay; $x$ is characteristics that determine the probability of discrete outcome for pedestrian $n ; \beta_{p}$ is estimating parameter; $p$ is number of independent variables.

$80 \%$ of pedestrian data were used for pedestrianvehicular interaction model calibration and remaining $20 \%$ data were used for model validation. To determine influence factors of pedestrian-vehicular interaction, a Pearson's correlation analysis was calculated between ten parameters by SPSS 16.0 and identified four factors had significant effects and results are shown in Table 3. Pedestrian crossing behaviour has been studied in this section under the collected field data. These results would be used in the development of the proposed models mentioned in the following section.

Table 3. List of parameters of pedestrian-vehicular interaction behaviour and results of Pearson's correlation analysis

\begin{tabular}{|l|l|c|c|}
\hline \multicolumn{1}{|c|}{ Variable } & \multicolumn{1}{|c|}{ Description } & $\begin{array}{c}\text { Correlation } \\
\text { coefficient }\end{array}$ & Significant \\
\hline $\begin{array}{l}\text { Delay due to pedestrian- } \\
\text { vehicular interaction }\end{array}$ & 0 for not receiving delay and 1 for receiving delay & 1 & - \\
\hline Pedestrian gender & 0 for male and 1 for female & $-0.010^{\mathrm{b}}$ & $0.802^{\mathrm{b}}$ \\
\hline Pedestrian age group & 0 for old pedestrian and 1 for adult pedestrian & $-0.006^{\mathrm{b}}$ & $0.887^{\mathrm{b}}$ \\
\hline No. of pedestrian & 0 for more than one pedestrian and 1 for single pedestrian & $0.016^{\mathrm{b}}$ & $0.690^{\mathrm{b}}$ \\
\hline Pedestrian group size & Number of persons composing the pedestrian group & $0.111^{\mathrm{a}}$ & $0.007^{\mathrm{a}}$ \\
\hline Pedestrian traffic signal & 0 for pedestrian green phase and 1 for non-green phase & $0.232^{\mathrm{a}}$ & $0.000^{\mathrm{a}}$ \\
\hline $\begin{array}{l}\text { Pedestrian direction of } \\
\text { movement }\end{array}$ & 0 for downstream to upstream and 1 for upstream to downstream & $-0.011^{\mathrm{b}}$ & $0.792^{\mathrm{b}}$ \\
\hline $\begin{array}{l}\text { Time difference between } \\
\text { pedestrian entering crosswalk } \\
\text { and vehicle arriving crosswalk }\end{array}$ & $\begin{array}{l}\text { Time difference between pedestrian start walk from waiting area to } \\
\text { crosswalk width and first moving vehicle reach crosswalk width }\end{array}$ & $-0.598^{\mathrm{a}}$ & $0.000^{\mathrm{a}}$ \\
\hline Number of interacting vehicles & $\begin{array}{l}\text { Number of vehicles pedestrian facing in crosswalk after entering } \\
\text { crosswalk width }\end{array}$ & $0.429^{\mathrm{a}}$ & $0.000^{\mathrm{a}}$ \\
\hline Pedestrian speed & Pedestrian crossing speed & $0.043^{\mathrm{b}}$ & $0.291^{\mathrm{b}}$ \\
\hline
\end{tabular}

Notes: ${ }^{a}$ correlation is significant; ${ }^{b}$ correlation is not significant (at $99 \%$ of confidence level); ${ }^{*}$ children age group data is not sufficient to model. 


\section{Proposed Pedestrian Delay Models}

Delay is a measure of effectiveness parameter for determining LOS at signalized intersections. New methodology has been proposed for estimating average pedestrian delay at two different kinds of signalized intersections. They differ from each other in the factor of pedestrianvehicular interactions. First model estimates pedestrian delay based on pedestrian compliance behaviour and the second model estimates based on pedestrian noncompliance behaviour with vehicular interaction at signalized intersections.

\subsection{Modelling Pedestrian Delay Based on Pedestrian Compliance Behaviour}

First model is developed, based on data represent pedestrians' compliance with traffic signal and no pedestrianvehicular interactions. This delay model has two components, which are the average waiting time delay, and the crossing time delay. Incorporating correction factor for non-uniform arrival pattern and crossing speed variations, the new model is developed and it as follows:

$$
D_{P C}=D_{W T}+D_{C T},
$$

where: $D_{P C}$ is overall average pedestrian delay at signalized intersections; $D_{W T}$ is average waiting time delay during non-green phases per pedestrian; $D_{C T}$ is average crossing time delay per pedestrian.

\section{Waiting Time Delay}

Pedestrian arrival pattern has been found to be non-uniform. HCM (2010) waiting time delay (Eq. (1)) model which relies on the assumption of uniform arrival rate was modified to account for the non-uniform arrival rate under mixed traffic conditions by including the correction factor (Eq. (6)) as follows:

$$
D_{W T}=\frac{\alpha_{1} \cdot(C-G)^{2}}{2 \cdot C},
$$

where: value of $(C-G)$ is the effective red time; $\alpha_{1}$ is correction factor for non-uniform arrival rate and this value is calculated from Eq. (7); $C$ is cycle time; $G$ is pedestrian green time.

\section{Crossing Time Delay}

Pedestrian crossing time delay is time difference between actual field crossing time and ideal crossing time. Ideal crossing time is the ratio between crosswalk length and pedestrian 15th percentile crossing speed. A simple method is introduced to calculate crossing time delay accurately with less time involved. From various field data, actual crossing time and ideal crossing time were calculated. A new correction factor for pedestrian actual field crossing time $\gamma$ is introduced and defined as the ratio between actual crossing time and ideal crossing time:

$$
\begin{aligned}
& \gamma=\frac{t_{A}}{t_{I}} ; \\
& t_{A}=\gamma \cdot t_{I},
\end{aligned}
$$

where: $t_{A}$ is pedestrian actual field crossing time; $t_{I}$ is average pedestrian ideal crossing time, $t_{I}=\frac{L}{V_{15}} ; L$ is length of crosswalk; $V_{15}$ is 15 th percentile crossing speed; $\gamma$ is correction factor for pedestrian actual field crossing time.

The linear relationship between $V_{15}$ and correction factor for pedestrian actual field crossing time $\gamma$ on crosswalk was developed by using all observed field data and it can be expressed by:

$$
\gamma=0.0168 \cdot V_{15}+1.0225 \text {. }
$$

Pedestrian crossing time delay can be calculated by:

$$
D_{C T}=t_{A}-t_{I}=\gamma \cdot t_{I}-t_{I}=(\gamma-1) \cdot t_{I} \text {. }
$$

Using the $V_{15}$ value from the field data, $\gamma$-value can be calculated based on Eq. (14). Pedestrian crossing delay has been calculated with ideal crossing time and the correction factor $\gamma$. This method of estimation is simple, quick and more accurate. Final pedestrian delay model based on pedestrian compliance behaviour is as follows:

$$
D_{P C}=\frac{\alpha_{1} \cdot(C-G)^{2}}{2 \cdot C}+(\gamma-1) \cdot t_{I},
$$

where: $D_{P C}$ is overall average pedestrian delay at signalized intersections. The developed delay model was different from existing models in terms of incorporating arrival pattern and crossing speed variations. This model can be useful in assessing the performance and adequacy of existing signalized intersections, developing appropriate crosswalk design and LOS at signalized intersections.

\subsection{Modelling Pedestrian Delay based on Noncompliance Behaviour with Vehicular Interaction}

The second pedestrian delay model is developed based on the pedestrian non-compliance behaviour by considering pedestrian-vehicular interaction. This model is applicable where pedestrian noncompliance is predominant. This model has three components. First component is average waiting time delay considering pedestrian non-compliance, second component is crossing time delay, and third component is the pedestrian-vehicular interaction delay. The proposed new model is described as follows:

$$
D_{P N C}=D_{W T \_N C}+D_{C T}+D_{P V I T} \text {, }
$$

where: $D_{P N C}$ is overall average pedestrian delay at signalized intersections based on pedestrian noncompliance behaviour and vehicular interaction; $D_{W T_{-} N C}$ is average waiting time delay during non-green phases per pedestrian considering pedestrian non-compliance rate; $D_{C T}$ is average crossing time delay per pedestrian; $D_{P V I T}$ is average pedestrian-vehicular interaction time delay during pedestrian green and non-green phases per pedestrian.

\section{Waiting Time Delay with Pedestrian Non-Compliance}

Pedestrians non-complying with the traffic signals are utilizing the effective red time without waiting at the waiting areas in signalized intersections. During estimation of pedestrian waiting time delay, utilized red time by 
noncompliant pedestrians is reduced from effective red time. So, the value of effective red time $\left(C-\left(G+\alpha_{2} \cdot R\right)\right)$ was calculated based on pedestrian non-compliance rate and by also utilising the modified form of HCM waiting time delay model. This model is described as follows:

$$
D_{W T_{-} N C}=\frac{\alpha_{1} \cdot\left(C-\left(G+\alpha_{2} \cdot R\right)\right)^{2}}{2 \cdot C},
$$

where: $\alpha_{2}$ is percentage of pedestrian start crossing during non-green phases; $R$ is pedestrian red time; $\alpha_{1}$ is value calculated by using Eq. (7). If the pedestrians are comply with traffic signal, the value of $\alpha_{2}$ becomes zero in waiting time delay estimation. Crossing time delay $D_{C T}$ is calculated by using same method explained under Section 6.1 using Eq. (15).

\section{Modelling Pedestrian-Vehicular Interaction Delay}

While signal non-complying, the pedestrians interact with vehicles in the crosswalk or at the median and receive waiting time delay. These influencing factors of pedestrian-vehicular interaction delay are identified by conducting correlation coefficient test and also using the results from Table 3. Based on these four significant factors, pedestrian-vehicular interaction delay model is developed. The probability of receiving delay in crosswalk is based on utility function and the model is mentioned in Eq. (19). A BL model was developed in NLOGIT 4 software (http://www.limdep.com) by using $80 \%$ of pedestrian samples and the model is described as follows:

$$
\begin{aligned}
& X=g(x)=4.0988+1.1905 \cdot x_{1}+ \\
& 1.3687 \cdot x_{2}-0.7988 \cdot x_{3}+0.6802 \cdot x_{4},
\end{aligned}
$$

where: $x_{1}$ is pedestrian platoon size $(t=3.112, p=0.001)$; $x_{2}$ is pedestrian traffic signal $(t=3.180, p=0.001) ; x_{3}$ is time difference between pedestrian enter crosswalk and first vehicle arriving crosswalk $(t=-8.559, p=0.000) ; x_{4}$ is number of interacting vehicles $(t=6.359, p=0.000)$. The probability of a pedestrian receiving delay at a crosswalk is calculated from Eq. (8). Binary logit model variables are significant in $p$-value $(<0.01)$ and $t$-value (>2.58) statistical test.

Accurately predicted value is $94.43 \%$ with 0.7331 and 0.8183 values of McFadden pseudo $R$-squared and Estrella $R$-values respectively. Therefore, the developed binary logit model could provide a good prediction for pedestrian-vehicular interaction delay at crosswalk. The linear relationship between probabilities of pedestrians interact with vehicles $P$ and average pedestrians-vehicular interaction delay $D_{P V I T}$ at crosswalks were developed and it can be expressed by:

$$
D_{P V I T}=11.189 \cdot P-1.0713, R^{2}=0.97 \text {. }
$$

By using equation the average pedestrian-vehicular interaction delay has been calculated based on probability of pedestrian interact with vehicle in crosswalk. The developed delay model is as follows:

$$
\begin{aligned}
& D_{P N C}=\frac{\alpha_{1} \cdot\left(C-\left(G+\alpha_{2} \cdot R\right)\right)^{2}}{2 \cdot C}+ \\
& (\gamma-1) \cdot t+(11.189 \cdot P-1.0713),
\end{aligned}
$$

where: $D_{P N C}$ is overall average pedestrian delay at signalized intersections based on pedestrian noncompliance behaviour and vehicular interaction.

This model can be useful when evaluating the quality of pedestrian flow, providing safe and adequate crosswalks, estimating accurate pedestrian delay and LOS for local conditions, which is representative of the prevailing pedestrian condition.

\subsection{Validation of Developed Delay Models}

From collected data, $80 \%$ of training data were utilized for development of pedestrian delay models and remaining $20 \%$ data were utilized for validating the developed models. Individual delay components and overall average pedestrian delay values of both models were estimated and compared with measured field component's delay values for training data and validation data and shown in Table 4.

Table 4 shows that the estimated values were close to field values. The minimum difference between the values proves that the new models have high accuracy in estimation of actual pedestrian delay.

\subsection{Comparison of Developed Models with Existing Models}

Statistical performance level of proposed models were analysed by using Origin Pro 9 software (http://www. originlab.com/originpro) and compared with existing models. Mean Absolute Percentage Error (MAPE), Root Mean Square Error (RMSE), coefficient of correlation (Pearson's $R$ ), and coefficient of determination $R^{2}$ were compared between values from proposed models and existing models with field data and results are shown in Table 5 for training data and validation data. From Table 5, HCM (2010) model estimation delay values were higher than the field delay values, since non-uniform arrival rate and crossing speed variations of pedestrian were not considered in these models whereas the estimated values by developed pedestrian delay model based on compliance behaviour are much closer to field value. MAPE and RMSE error values of proposed pedestrian delay model are very less compared to the HCM (2010) model. So, the proposed new pedestrian delay model is based on compliance behaviour has more advantages than the existing models, because it is considering nonuniform pedestrian arrival pattern by establishing $\alpha_{1}$, and crossing speed variation by introducing $\gamma$.

Braun and Roddin (1978), Li et al. (2005b), Yang et al. (2005), and Nagraj and Vedagiri (2013) models predict higher delay values than the field value due to consideration of delay reduction due to pedestrian noncompliance rate and not considering pedestrian-vehicular interaction delay.

The existing models fail to predict the field delay accurately. The estimated value of developed delay model based on pedestrian noncompliance behaviour with vehicular interaction was reasonably closer to field delay indicating that this model has better estimation than other models with 0.982 coefficient of determination. 
Table 4. Comparison of proposed models values with field data

\begin{tabular}{|c|c|c|c|c|c|c|c|c|c|c|c|}
\hline \multirow[t]{2}{*}{ Data set } & \multirow[t]{2}{*}{ Model } & \multirow[t]{2}{*}{ Location } & \multicolumn{2}{|c|}{$\begin{array}{l}\text { Waiting time delay } \\
\text { per pedestrian }[\mathrm{s}]\end{array}$} & \multicolumn{2}{|c|}{$\begin{array}{l}\text { Crossing time delay } \\
\text { per pedestrian }[\mathrm{s}]\end{array}$} & \multicolumn{2}{|c|}{$\begin{array}{l}\text { Interaction delay } \\
\text { per pedestrian }[\mathrm{s}]\end{array}$} & \multicolumn{2}{|c|}{$\begin{array}{l}\text { Overall average } \\
\text { delay per } \\
\text { pedestrian }[\mathrm{s}]\end{array}$} & \multirow[t]{2}{*}{$\%$ difference } \\
\hline & & & Model & Field & Model & Field & Model & Field & Model & Field & \\
\hline \multirow{8}{*}{$\begin{array}{l}\text { Training } \\
\text { data }\end{array}$} & \multirow{4}{*}{1} & $A^{c}$ & 42.49 & 41.06 & 1.02 & 1.31 & - & - & 43.51 & 42.37 & 2.7 \\
\hline & & $\mathrm{B}^{\mathrm{c}}$ & 30.91 & 27.28 & 0.48 & 0.53 & - & - & 31.39 & 27.81 & 12.8 \\
\hline & & $\mathrm{C}^{\mathrm{c}}$ & 39.41 & 37.19 & 0.67 & 1.28 & - & - & 40.08 & 38.47 & 4.2 \\
\hline & & $\mathrm{D}^{\mathrm{c}}$ & 33.68 & 34.47 & 0.96 & 1.10 & - & - & 34.64 & 35.57 & 2.6 \\
\hline & \multirow{4}{*}{2} & $\mathrm{~A}^{\mathrm{nc}}$ & 23.16 & 24.89 & 1.02 & 0.61 & 8.21 & 7.81 & 32.39 & 33.31 & 2.8 \\
\hline & & $\mathrm{B}^{\mathrm{nc}}$ & 9.73 & 9.16 & 0.72 & 0.92 & 2.77 & 3.71 & 13.22 & 13.79 & 4.1 \\
\hline & & $\mathrm{C}^{\mathrm{nc}}$ & 2.71 & 1.96 & 0.66 & 0.36 & 10.12 & 12.49 & 13.49 & 14.80 & 8.8 \\
\hline & & $\mathrm{D}^{\mathrm{nc}}$ & 11.27 & 12.05 & 0.91 & 0.74 & 7.51 & 5.62 & 19.69 & 18.41 & 6.9 \\
\hline \multirow{6}{*}{$\begin{array}{l}\text { Validation } \\
\text { data }\end{array}$} & \multirow{3}{*}{1} & $A^{c}$ & 31.06 & 32.70 & 0.59 & 0.59 & - & - & 31.65 & 33.29 & 5.2 \\
\hline & & $\mathrm{C}^{\mathrm{c}}$ & 56.02 & 59.29 & 0.69 & 0.81 & - & - & 56.72 & 60.08 & 5.9 \\
\hline & & $\mathrm{D}^{\mathrm{c}}$ & 23.96 & 22.33 & 0.88 & 0.98 & - & - & 23.96 & 23.31 & 2.7 \\
\hline & \multirow{3}{*}{2} & $\mathrm{~A}^{\mathrm{nc}}$ & 9.644 & 10.86 & 0.95 & 0.86 & 8.53 & 10.01 & 19.13 & 21.78 & 13.6 \\
\hline & & $\mathrm{B}^{\mathrm{nc}}$ & 9.78 & 9.46 & 1.08 & 0.75 & 1.80 & 2.4 & 12.66 & 12.61 & 0.4 \\
\hline & & $\mathrm{C}^{\mathrm{nc}}$ & 7.89 & 7.143 & 1.06 & 1.30 & 6.5 & 7.33 & 15.45 & 15.77 & 2.1 \\
\hline
\end{tabular}

Notes: ${ }^{c}$ represent the data based on pedestrian compliance behaviour and results made for Model 1; ${ }^{\text {nc }}$ represent the data based on pedestrian noncompliance behaviour and interaction and results made for Model 2.

Table 5. Comparison of performance level of proposed model with existing model

\begin{tabular}{|c|c|c|c|c|c|c|c|c|}
\hline \multirow[b]{2}{*}{ Data set } & \multirow[b]{2}{*}{ Location } & \multicolumn{7}{|c|}{ Delay values [s/ped] } \\
\hline & & $\begin{array}{c}\text { Field } \\
\text { values }\end{array}$ & $\begin{array}{c}\text { HCM } \\
\text { (Eq. (1)) }\end{array}$ & $\begin{array}{c}\text { Proposed } \\
\text { Model } 1\end{array}$ & $\begin{array}{l}\text { Braun-Rodin } \\
\quad \text { (Eq. (2)) }\end{array}$ & $\begin{array}{l}\text { Qingfing } \\
(\text { Eq. (4)) }\end{array}$ & $\begin{array}{l}\text { Nagraj } \\
\text { (Eq. (5)) }\end{array}$ & $\begin{array}{c}\text { Proposed } \\
\text { Model } 2\end{array}$ \\
\hline \multirow{8}{*}{ Training data } & $\mathrm{A}^{\mathrm{c}}$ & 42.37 & 51.25 & 43.51 & - & - & - & - \\
\hline & $\mathrm{B}^{\mathrm{c}}$ & 27.81 & 40.78 & 31.39 & - & - & - & - \\
\hline & $\mathrm{C}^{\mathrm{c}}$ & 38.47 & 53.55 & 40.08 & - & - & - & - \\
\hline & $\mathrm{D}^{\mathrm{c}}$ & 35.57 & 24.09 & 34.64 & - & - & - & - \\
\hline & $\mathrm{A}^{\mathrm{nc}}$ & 33.31 & - & - & 33.62 & 27.01 & 18.53 & 32.39 \\
\hline & $\mathrm{B}^{\mathrm{nc}}$ & 13.79 & - & - & 23.09 & 21.19 & 17.43 & 13.22 \\
\hline & $\mathrm{C}^{\mathrm{nc}}$ & 14.80 & - & - & 48.12 & 37.97 & 17.71 & 13.49 \\
\hline & $\mathrm{D}^{\mathrm{nc}}$ & 18.41 & - & - & 20.79 & 26.04 & 9.65 & 19.69 \\
\hline \multirow{6}{*}{$\begin{array}{l}\text { Validation } \\
\text { data }\end{array}$} & $\mathrm{A}^{\mathrm{c}}$ & 33.29 & 51.25 & 31.65 & - & - & - & - \\
\hline & $\mathrm{C}^{\mathrm{c}}$ & 60.08 & 53.55 & 56.72 & - & - & - & - \\
\hline & $\mathrm{D}^{\mathrm{c}}$ & 23.31 & 24.09 & 23.96 & - & - & - & - \\
\hline & $\mathrm{A}^{\mathrm{nc}}$ & 21.73 & - & - & 30.91 & 26.02 & 24.42 & 19.13 \\
\hline & $\mathrm{B}^{\mathrm{nc}}$ & 12.61 & - & - & 27.13 & 27.93 & 23.05 & 12.66 \\
\hline & $\mathrm{C}^{\mathrm{nc}}$ & 15.77 & - & - & 11.28 & 23.31 & 10.61 & 15.45 \\
\hline \multirow{4}{*}{$\begin{array}{l}\text { Performance } \\
\text { level for } \\
\text { training data }\end{array}$} & MAPE & - & 0.342 & 0.056 & 0.766 & 0.676 & 0.345 & 0.057 \\
\hline & RMSE & - & 12.31 & 2.096 & 17.33 & 13.13 & 8.900 & 1.064 \\
\hline & Pearson's $R$ & - & 0.423 & 0.9569 & 0.033 & 0.102 & 0.232 & 0.992 \\
\hline & $R^{2}$ & - & 0.179 & 0.9156 & 0.001 & 0.012 & 0.054 & 0.984 \\
\hline \multirow{4}{*}{$\begin{array}{l}\text { Performance } \\
\text { level for } \\
\text { validation } \\
\text { data }\end{array}$} & MAPE & - & 0.227 & 0.044 & 0.619 & 0.312 & 0.427 & 0.048 \\
\hline & RMSE & - & 11.04 & 2.192 & 10.25 & 10.17 & 6.903 & 1.513 \\
\hline & Pearson's $R$ & - & 0.757 & 0.999 & 0.349 & 0.246 & 0.262 & 0.995 \\
\hline & $R^{2}$ & - & 0.574 & 0.998 & 0.755 & 0.879 & 0.863 & 0.982 \\
\hline
\end{tabular}

Notes: ${ }^{c}$ proposed model 1 represent compliance behaviour; ${ }^{\mathrm{nc}}$ proposed model 2 represent noncompliance behaviour with interaction. 
Therefore, the proposed new pedestrian delay model based on pedestrian noncompliance behaviour with vehicular interaction has few more advantages over the existing models, such as considering pedestrian behaviour by introducing non-compliance factor $\alpha_{2}$, and considering traffic conditions by establishing BL model for pedestrian-vehicular interaction. Finally, the performance results of proposed models provide more precise and reliable solutions.

\section{Conclusions}

It has been verified in this paper that the estimates of pedestrian delay from the existing models do not always match with the reality under mixed traffic conditions. In view of this, the authors have proposed and developed two new delay models for calculating pedestrian delay at signalized intersections by considering pedestrian crossing behaviour under mixed traffic conditions. Based on conducted video-graphic survey at a typical four arm, signalized intersections in Mumbai (India), pedestrian crossing behaviour have been analysed and the following conclusions are drawn.

Pedestrian gender, age and platoon had significant effect on pedestrian crossing speed variations. The design crossing speed is varying from $0.95 \mathrm{~m} / \mathrm{s}$ to $1.12 \mathrm{~m} / \mathrm{s}$ and the correction factor for pedestrian actual crossing time was introduced.

The rate of pedestrian noncompliance was found to be $57.29 \%$ in the selected sites. The rate of noncompliance of male pedestrian is more than female pedestrian. The reasons were to reduce more waiting time delay, less pedestrian green time, less vehicle flow and absent of traffic assistants.

BL model was developed with significant parameters for finding the probability of pedestrian receiving vehicular interaction delay in crosswalks. Accurately predicted value is $94.4 \%$ with $0.733 \mathrm{McFadden}$ pseudo $R$-squared values. The linear relation between probability and average pedestrian interaction delay was developed with $0.97 R$-square value at crosswalk.

Based on crossing speed variation and non-uniform arrival rate, a new pedestrian delay model based on compliance behaviour has been developed for delay estimation. Considering crossing speed variation, arrival rate, pedestrian non-compliance rate and interactions behaviour, another new pedestrian delay model based on noncompliance behaviour with vehicular interaction has been developed. Both models were validated with field data and percentage of difference is less. Finally, compared with existing models, and the results indicate that the new models can provide more accurate and reliable solutions with less error. Validate the developed models with data from other cities as future scope of the present work.

\section{Recommendations}

This study is expected to help traffic engineers and policy makers understand pedestrian crossing behaviour and delay model under mixed traffic conditions at signalized intersections. The authors opine that the developed new pedestrian delay model based on compliance behaviour is applicable to traffic conditions of developed and developing countries and useful when optimizing traffic signal control and evaluating pedestrian LOS at signalized intersections but when the pedestrian-vehicular interactions occur, the model fails to estimate delay accurately.

To overcome this shortcoming, the new pedestrian delay model based on pedestrian noncompliance behaviour with vehicular interaction is developed and is best suitable for developing countries like India where pedestrian noncompliance are predominant and pedestrian-vehicular interaction are frequent. This model can be useful when evaluating the quality of pedestrian flow, designing facilities, providing safe and adequate crosswalks at signalized intersections. The developed delay model based on noncompliance and interaction is representing the prevailing pedestrian situation, which help policy makers to take suitable corrective and management measures.

\section{References}

Al-Azzawi, M.; Raeside, R. 2008. Modeling pedestrian walking speeds on sidewalks, Journal of Urban Planning and Development 133(3): 211-219.

http://dx.doi.org/10.1061/(ASCE)0733-9488(2007)133:3(211)

Braun, R. R.; Roddin, M. F. 1978. Quantifying the Benefits of Separating Pedestrians and Vehicles. NCHRP Report No 189. Transportation Research Board, US. 127 p.

Chen, J.; Shi, J.-J.; Li, X.-L.; Zhao, Q. 2011. Pedestrian behavior and traffic violations at signalized intersections, in ICCTP 2011: Towards Sustainable Transportation Systems, 14-17 August 2011, Nanjing, China, 1103-1110. http://dx.doi.org/10.1061/41186(421)108

Chen, K.-M.; Luo, X.-Q.; Ji, H.; Zhao, Y.-D. 2010. Towards the pedestrian delay estimation at intersections under vehicular platoon caused conflicts, Scientific Research and Essays 5(9): 941-947.

Gates, T.; Noyce, D.; Bill, A.; Van Ee, N. 2006. Recommended walking speeds for timing of pedestrian clearance intervals based on characteristics of the pedestrian population, Transportation Research Record: Journal of the Transportation Research Board 1982: 38-47. http://dx.doi.org/10.3141/1982-07

Huang, C.; Ma, W. 2010. A statistical analysis of pedestrian speed on signalized intersection crosswalk, in ICCTP 2010: Integrated Transportation Systems: Green, Intelligent, Reliable, 4-8 August 2010, Beijing, China, 1401-1407. http://dx.doi.org/10.1061/41127(382)151

HCM. 2010. Highway Capacity Manual. Transportation Research Board. 5th edition. $1650 \mathrm{p}$.

IRC: 103-2012. Guidelines for Pedestrian Facilities. Indian Roads Congress.

Jiang, N.; Shi, M.; Xiao, Y., Shi, K.; Watson, B. 2011. Factors affecting pedestrian crossing behaviors at signalized crosswalks in urban areas in Beijing and Singapore, in 1st International Conference on Transportation Information and Safety 2011 (ICTIS 2011), 30 June - 2 July 2011, Wuhan, China, 2: 1089-1096.

Kruszyna, M.; Mackiewicz, P.; Szydlo, A. 2006. Influence of pedestrians' entry process on pedestrian delays at signalcontrolled crosswalks, Journal of Transportation Engineering 132(11): 855-861.

http://dx.doi.org/10.1061/(ASCE)0733-947X(2006)132:11 (855) 
Lam, W. H. K.; Lee, J. Y. S.; Cheung, C. Y. 2002. A study of the bi-directional pedestrian flow characteristics at Hong Kong signalized crosswalk facilities, Transportation 29(2): 169-192. http://dx.doi.org/10.1023/A:1014226416702

Li, Q.; Wang, Z.; Yang, J. 2005a. A numerical model for estimating pedestrian delays at signalized intersections in developing cities, in J. Zhang, J.-H. He, Y. Fu (Eds.). Computational and Information Science, 419-424. http://dx.doi.org/10.1007/978-3-540-30497-5_65

Li, Q.; Wang, Z.; Yang, J.; Wang, J. 2005b. Pedestrian delay estimation at signalized intersections in developing cities, Transportation Research Part A: Policy and Practice 39(1): 61-73. http://dx.doi.org/10.1016/j.tra.2004.11.002

Li, Y.-F.; Shi, Z.-K.; Zhou, Z.-N. 2009. Estimation of probability of swarming pedestrians violation at signalized intersections in developing cities, Journal of Southwest Jiaotong University (1): 80-85.

Ling, Z.; Ni, Y.; Li, K. 2012. Modeling interactions between pedestrians and right-turn vehicles at signalized intersections, CICTP 2012: Multimodal Transportation Systems: Convenient, Safe, Cost-Effective, Efficient, 3-6 August 2012, Beijing, China, 2072-2084.

http://dx.doi.org/10.1061/9780784412442.211

Marisamynathan, S.; Vedagiri, P. 2013. Modeling pedestrian delay at signalized intersection crosswalks under mixed traffic condition, Procedia - Social and Behavioral Sciences 104: 708-717. http://dx.doi.org/10.1016/j.sbspro.2013.11.165

Mousa, R. M. 2002. Analysis and modeling of measured delays at isolated signalized intersections, Journal of Transportation Engineering 128(4): 347-354.

http://dx.doi.org/10.1061/(ASCE)0733-947X(2002)128:4(347)

Nagraj, R.; Vedagiri, P. 2013. Modeling pedestrian delay and level of service at signalized intersection crosswalks under mixed traffic conditions, Transportation Research Record: Journal of the Transportation Research Board 2394: 70-76. http://dx.doi.org/10.3141/2394-09

Oh, H.; Sisiopiku, V. P. 2000. Probabilistic models for pedestrian capacity and delay at roundabouts, in Fourth International Symposium on Highway Capacity, 27 June - 1 July 2000, Maui, Hawaii, 459-470.

Olszewski, P. 1993. Overall delay, stopped delay, and stops at signalized intersections, Journal of Transportation Engineering 119(6): 835-852.

http://dx.doi.org/10.1061/(ASCE)0733-947X(1993)119:6(835)

Rahman, K.; Ghani, N. A.; Kamil, A. A.; Mustafa, A. 2012. Analysis of pedestrian free flow walking speed in a least developing country: a factorial design study, Research Journal of Applied Sciences, Engineering and Technology 4(21): 4299-4304.

Rastogi, R.; Chandra, S.; Vamsheedhar, J.; Das, V. R. 2011. Parametric study of pedestrian speeds at midblock crossings, Journal of Urban Planning and Development 137(4): 381-389. http://dx.doi.org/10.1061/(ASCE)UP.1943-5444.0000083

Virkler, M. 1998. Pedestrian compliance effects on signal delay, Transportation Research Record: Journal of the Transportation Research Board 1636: 88-91. http://dx.doi.org/10.3141/1636-14

Wang, X.; Tian, Z. 2010. Pedestrian delay at signalized intersections with a two-stage crossing design, Transportation Research Record: Journal of the Transportation Research Board 2173: 133-138. http://dx.doi.org/10.3141/2173-16

Xie, D.-F.; Gao, Z.-Y.; Zhao, X.-M.; Wang, D. Z. W. 2012. Cellular automaton modeling of the interaction between ve- hicles and pedestrians at signalized crosswalk, Journal of Transportation Engineering 138(12): 1442-1452.

http://dx.doi.org/10.1061/(ASCE)TE.1943-5436.0000462

Yang, J.; Li, Q.; Wang, Z.; Wang, J. 2005. Estimating pedestrian delays at signalized intersections in developing cities by Monte Carlo method, Mathematics and Computers in Simulation 68(4): 329-337. http://dx.doi.org/10.1016/j.matcom.2005.01.017

Zhou, Z.-P.; Ren, G.; Wang, W. 2011. Modeling violations of pedestrian road-crossing behavior at signalized intersections, in ICCTP 2011: Towards Sustainable Transportation Systems, 14-17 August 2011, Nanjing, China, 2490-2499. http://dx.doi.org/10.1061/41186(421)250 